\title{
e-Migrinter
}

$8 \mid 2012$

Regards sur les migrations sud-asiatiques

\section{Regards sur les migrations sud-asiatiques}

Aurélie Varrel et Philippe Venier

\section{(2) OpenEdition}

Journals

Édition électronique

URL : https://journals.openedition.org/e-migrinter/579

DOl : $10.4000 /$ e-migrinter. 579

ISSN : 1961-9685

Éditeur

UMR 7301 - Migrinter

Édition imprimée

Date de publication : 4 avril 2012

Pagination : 03-05

ISSN : 1961-9685

\section{Référence électronique}

Aurélie Varrel et Philippe Venier, « Regards sur les migrations sud-asiatiques », e-Migrinter [En ligne], 8|

2012, mis en ligne le, consulté le 20 mai 2021. URL : http://journals.openedition.org/e-migrinter/579 DOI : https://doi.org/10.4000/e-migrinter.579 


\title{
ÉDITORIAL
}

\section{Regards sur les migrations sud-asiatiques}

\author{
Aurélie Varrel \& Philippe Venier
}

C

e huitième numéro de la revue e-migrinter est issu du séminaire international « Les migrations internationales sudasiatiques » qui s'est tenu à la Maison des Sciences de 1'Homme et de la Societé de Poitiers les 27 et 28 avril 2010. Les contributions réunies dans ce dossier nous invitent à décentrer nos regards en s'intéressant à un bassin migratoire majeur, le bassin sudasiatique, inscrit dans la « mondialisation migratoire " et pourtant encore mal connu.
Cette livraison propose d'analyser les dynamiques migratoires sud-asiatiques. Elles concernent des populations et des espaces de départ qui ont été encore peu explorés par la recherche française alors qu'il existe une production scientifique importante concernant ces populations en diaspora en Amérique du Nord et au Royaume Uni. Les circulations et les pratiques transnationales des migrants sud-asiatiques montrent que d'autres espaces migratoires se sont constitués: le Golfe arabo-persique, l'Europe ou l'Asie du Sud-Est par exemple.

Le bassin migratoire indien, à l'origine d'une émigration internationale massive, est aussi le lieu d'importantes migrations internes et d'immigrations à l'échelle régionale. Ces migrations vers et en Inde montrent qu'elles s'inscrivent dans bien des cas dans la mondialisation migratoire : ainsi, les stratégies migratoires mises en œuvre par les migrants à destination des pays du Golfe arabo-persique et/ou de l'Occident commencent bien souvent par une migration à l'intérieur du sous-continent.

Les différents articles publiés dans ce numéro sont le résultat de travaux menés par des chercheurs français et indiens. $\mathrm{Ne}$ prétendant nullement à l'exhaustivité, ils révèlent néanmoins des logiques migratoires contemporaines. L'Asie du Sud est en effet pleinement insérée dans la globalisation des migrations internationales. Il suffit de constater l'importance en nombre et en dispersion des diasporas sud-asiatiques, notamment en relation avec la période coloniale - l'Asie du Sud telle qu'on la conçoit correspondant peu ou prou à l'empire des Indes. Le poids démographique de cette région du monde en fait un vivier de 
main-d'œuvre (de non qualifiée à très qualifiée) essentiel pour le Golfe, mais également pour les économies occidentales. Par ailleurs, l'Inde, pays majeur de l'Asie du Sud et pays émergent à l'échelle internationale, devient elle-même un pôle ordonnant les dynamiques migratoires en relation avec les tensions géopolitiques de la région.

La question des réfugiés en Asie du Sud constitue un thème abordé dans la première partie du dossier. Les articles de Julie Baujard et d'Anthony GoreauPonceaud mettent en évidence le rôle central de l'Inde dans l'accueil des populations en exil en provenance de l'aire régionale. L'Inde, qui n'est pas encore signataire de la convention de Genève, n'accorde qu'un mandat limité au HCR à qui elle délègue - ou pas - la gestion des réfugiés selon les groupes concernés. Julie Baujard traite ainsi de la question des réfugiés urbains à Delhi (Afghans, Birmans et Tibétains). Elle analyse le traitement différencié que leur accorde l'Inde selon leur origine, leur appartenance communautaire et confessionnelle, mais également selon son agenda géopolitique. Anthony Goreau-Ponceaud, quant à lui, s'attache à décrire l'arrivée des Tamouls du Sri Lanka et de leur installation dans les camps de l'Inde du Sud. Il présente ces camps comme des "antimondes» qui sont autant d'étapes sur les «antiroutes» de la diaspora.

La deuxième partie réunit des contributions centrées sur un des pôles majeurs de l'immigration sud-asiatique, le Golfe arabo-persique, à partir de l'exemple du Qatar.

Revisitant les clichés sur les Indiens dans le Golfe, Radhika Kanchana décrit l'évolution des profils et des projets migratoires: l'augmentation du nombre de migrants très qualifiés parallèlement à un allongement de la durée en migration se traduisent par une visibilité économique et sociale croissante. L'auteure souligne aussi les nouvelles perspectives qui se présentent pour la deuxième génération.

La contribution de Tristan Bruslé décrit la situation des migrants népalais peu qualifiés, logés dans des compounds situés à l'écart des villes. Cette exploration dessine une géographie des espaces intimes des migrants dans le camp de travail. En outre, elle questionne le développement de comportements consuméristes qui se doublent de nouvelles pratiques d'ostentation et de mise en scène rendues possibles par leur appropriation des NTIC. Ces outils redéfinissent les liens avec la communauté népalaise au pays et en migration, dans leur forme comme dans leur contenu.

La troisième partie met l'accent sur de nouvelles configurations migratoires résolument inscrites dans la mondialisation. Deux figures de migrants qualifiés indiens émergent: les infirmières et les informaticiens. Les différentes contributions mettent en évidence de nouveaux parcours, de destinations nouvelles - ou d'autres qui sont réactivées - et des situations inédites qui questionnent les identités. Marie Percot nous offre une analyse originale du cheminement migratoire des infirmières indiennes jusqu'en Irlande. Désormais recherché par certains pays occidentaux, ce type de compétences professionnelles leur ouvre les portes d'une insertion économique et sociale après un passage obligé dans le Golfe qui peut ainsi être considéré comme un espace tremplin. Au prisme du genre, l'auteure choisit de se pencher sur les époux de ces professionnelles de santé. Au-delà des perspectives habituelles sur les hommes moteurs de la migration ou sur les "migrantes autonomes", elle explore le repositionnement des identités masculines pour les migrants accompagnants.

Éric Leclerc aborde le cas des informaticiens indiens envoyés à Kuala Lumpur (Malaisie) et dont la migration relève de la mondialisation des travailleurs 
hautement qualifiés. En analysant leur présence à Brickfields, vieux quartier indien de Kuala Lumpur, il s'interroge sur les liens qui se nouent - ou pas - à partir d'une appartenance ethnique commune. Il réinterroge également la frontière entre espaces urbains dits intégrés et exclus en fonction des pratiques résidentielles de cette catégorie socio-professionnelle.

L'ensemble de ces contributions ouvre sur des champs de recherche encore peu explorés. Le premier concerne les relations entre les réfugiés/étrangers résidant en Inde et les populations locales ainsi que les relations socio-économiques, culturelles et confessionnelles qui en découlent. La seconde piste de réflexion a trait aux nouvelles technologies de l'information et de la communication. Comment sont-elles utilisées comme instrument de repositionnement socio-économique dans le pays d'origine? En quoi ces outils participent-ils à un renforcement et/ou à une création d'espaces relationnels virtuels dans la communauté dans son ensemble?

Les différentes contributions publiées dans ce dossier nous alertent sur d'autres pistes de recherche qu'il convient de poursuivre : la question de la masculinité en migration d'abord, essentielle pour saisir les rapports de genre en migration; la question des réseaux transnationaux qui, d'une part, structurent les populations réfugiées et leur diaspora résidant dans les pays occidentaux, et, d'autre part, interrogent la place de l'Inde pour ces diasporas de l'exil en construction; la question de la mondialisation des flux migratoires sud-asiatiques, laquelle met en relation - et parfois en tension - des groupes diasporiques constitués à différentes époques avec des trajectoires économiques et migratoires diversifiées ; enfin, la question des fronts migratoires nouveaux et/ou réactivés - illustrés par le cas de la Malaisie. Ils constituent des espaces à explorer, que l'on pense par exemple à l'Afrique de l'Est où l'immigration indienne réapparait sur les traces d'anciennes installations coloniales, ou encore aux régions du monde concernées par le coolie trade. Au regard de la puissance régionale indienne qui s'affirme et de sa politique de soft power, l'étude de ces fronts offre de nouvelles perspectives de recherche.

Ce numéro se conclut par les deux rubriques classiques: vie du labo et notes de lecture. On lira une note de terrain proposée par Larry Becker, chercheur invité à Migrinter, puis un texte relatant le séjour de recherche dans notre laboratoire de Patrícia Tavares de Freita, doctorante brésilienne. Sont ensuite proposés deux résumés de thèses soutenues en 2011 à l'Université de Poitiers (Céline Bergeon et Marie Chabrol), ainsi que trois comptes rendus de séminaires et de conférences qui se sont tenus à la MSHS et à l'Espace Mendes-France. Pour finir, la présentation de cinq ouvrages dans le champ des migrations et de l'altérité clôt ce huitième numéro de e-migrinter.

Aurélie Varrel

Chargée de recherche en Géographie Centre d'Etudes de l'Inde et de l'Asie du Sud - UMR 8564 (CNRS-EHESS) École des Hautes Études en Sciences Sociales, Paris aurelie.varrel@,ehess.fr

Philippe Venier MCF en Géographie Université de Poitiers MIGRINTER - UMR 7301 philippe.venier@univ-poitiers.fr 